\section{Eqüidade no tempo de espera para determinadas cirurgias eletivas segundo o tipo de hospital em Sorocaba, SP}

\section{Equity in waiting time for some elective surgeries according to hospital type in Sorocaba, SP}

\section{Thiago Caldi de Carvalho'}

\section{Reinaldo José Gianini²}

${ }^{1}$ Acadêmico do $6^{\circ}$. ano de Medicina da Faculdade de Ciências Médicas - Centro de Ciências Médicas e Biológicas de Sorocaba - PUCSP

${ }^{2}$ Professor Titular do Departamento de Medicina da Faculdade de Ciências Médicas - Centro de Ciências Médicas e Biológicas de Sorocaba - PUCSP / Pesquisador do LIM-39 do HC/FMUSP

\section{Resumo}

Introdução: A eqüidade na atenção à saúde tem sido alvo de grande preocupação, incluindo países em desenvolvimento e desenvolvidos. A eqüidade horizontal, entendida como tratamento igual de indivíduos que se encontram na mesma situação de saúde, tem sido investigada nos diferentes níveis de atenção à saúde. Objetivos: $\mathrm{O}$ presente estudo busca verificar se existem disparidades no tempo de espera para cirurgias eletivas (safenectomia, colecistectomia, hemorroidectomia e histerectomia) segundo o tipo de hospital, público ou privado. Metodologia: Realizou-se, em Sorocaba, um estudo transversal com 40 pacientes atendidos por hospital privado e 40 atendidos por hospital público, no período de outubro e novembro de 2005. Além do tempo de espera e tipo de hospital, foram pesquisados sexo, idade, escolaridade, renda, situação conjugal, procedência, tipo de cirurgia realizada e presença de determinadas comorbidades. Resultados: Observou-se um tempo de espera maior nas categorias: hospital público ( 5,5 meses; $p<0,001)$, baixa escolaridade ( 3,5 meses; $\mathrm{p}<0,001)$, menor renda (Spearman $=-0,4426 ; \mathrm{p}<0,001$ ), procedência de outros municípios (2 meses; $\mathrm{p}=0,009$ ), e cirurgia de safenectomia (5 a 7 meses; $\mathrm{p}=0,04)$. Após o ajuste para as variáveis comorbidade, renda, escolaridade ou procedência, o tempo de espera no hospital público se manteve significantemente maior (diferença mínima de 4,93 meses quando ajustada por renda; IC95\% 3,4-6,4; $\mathrm{p}<0,001)$. Conclusão: Verifica-se relevante iniqüidade na atenção à saúde relacionada ao tipo de prestador de serviços de saúde.

Palavras chaves: Eqüidade. [Tempo de] espera. Cirurgia eletiva. Fatores sociais. Atenção à saúde. Políticas de saúde. 


\section{Abstract}

Introduction: Analyzing the waiting time for certain elective surgeries (saphenectomy, cholecystectomy, hemorrhoidectomy, and hysterectomy) according to hospital type, the present study intends to find if there are differences in health care. Methods: A crosssectional study was performed in Sorocaba with 40 individuals from a private hospital and 40 from a public hospital, interviewed in October and November 2005. Results: A longer waiting time was observed for the following categories: public hospital (5.5 months; $\mathrm{p}<0.001)$, lower education (3.5 months; $\mathrm{p}<0.001$ ), lower income (Spearman $=-0.4426 ; p<0.001$ ), residence out of Sorocaba ( 2 months; $\mathrm{p}=0.009$ ), and saphenectomy (5 to 7 months; $\mathrm{p}=0.04$ ). Adjusted for confounding, comorbidity, income, education, or residence variables, waiting time in public hospitals still was significantly longer (a difference of at least 4.93 months, when adjusted for income; 95\%CI 3.4-6.4; $\mathrm{p}<0.001)$. Conclusion: There was a relevant inequity in health care related mainly to the type of health care provider.

Keywords: Equity. Waiting time. Elective surgery. Social factors. Health care. Health policy.

\section{Introdução}

Quando nos referimos à eqüidade, logo vem à memória o conceito de igualdade, associação parcialmente correta porque eqüidade tem definição mais ampla: distribuição justa de determinado atributo populacional junto com a eficiência, a liberdade de escolha pelo consumidor e a maximização da saúde ${ }^{1}$. Segundo Pereira², "ao se definir eqüidade na prestação de serviços de saúde em termos de igualdade, o conceito envolve duas dimensões importantes: eqüidade horizontal - tratamento igual de indivíduos que se encontram numa situação de saúde igual; e eqüidade vertical - tratamento apropriadamente desigual de indivíduos em situações de saúde distintas".

Assim considerada, observamos que em nosso país ainda enfrentamos vários obstáculos à eqüidade. Por exemplo, ao se verificar que o gasto público em saúde como proporção do Produto Interno Bruto em 2000 no Brasil foi de $3,15 \%^{3}$ e que a Organização Mundial da Saúde preconiza como parâmetro a ser alcançado na estratégia da "Saúde para Todos no ano 2000" um percentual de $5 \%$ ou mais, isto significaria $58,73 \%$ acima do empregado no Brasil. Portanto, tendemos a cogitar que a escassez de verbas públicas poderá levar a uma deterioração estrutural do sistema de saúde público vigente, com a conseqüente fragilização de seu efeito de proteção social àqueles que mais dependem do Sistema Único de Saúde, e agravamento da iniqüidade ${ }^{5}$.

Realmente, isto tem sido uma preocupação constante de organismos internacionais, e não se restringe ao Brasil, estendendo-se a vários países. A Organização Pan-Americana de Saúde ${ }^{6}$ reconhece que contextos econômicos e sociais desempenham papel determinante na situação de saúde da população e propõe que sejam aumentados os esforços no sentido de caracterizar as desigualdades da situação sanitária das populações e do acesso aos serviços de saúde, identificando os grupos de maior risco para que medidas efetivas produzam um impacto de melhoria da saúde nas populações. 
Entre as várias possibilidades de estudo da eqüidade na atenção à saúde, temos as pesquisas que descrevem as disparidades ao nível da prevenção secundária. Esses estudos enfocam especificamente o Tratamento Imediato e a Limitação de Incapacidades ${ }^{1}$ , momento a partir do qual o diagnóstico já foi realizado e o paciente em questão já sofre com as repercussões daquele quadro clínico. Nesse sentido, trabalhos científicos sobre o tema têm sido realizados inclusive em países cujos Î́ndices de Desenvolvimento Humano estão entre os maiores do mun$\mathrm{do}^{7}$. Para isso analisam o tempo de espera de pacientes que realizaram cirurgias eletivas - cirurgias que podem ser programadas, não havendo necessidade de intervenção imediata $^{8}$.

O tempo de espera que é pesquisado pode se referir ao intervalo entre a realização do procedimento cirúrgico e a primeira consulta médica motivada pelo problema de saúde em questão, ou a consulta com o especialista, ou ainda a consulta médica que indicou a cirurgia ${ }^{9}$. É importante observar que um tempo de espera excessivo pode ter implicações desfavoráveis ${ }^{10}$, com repercussões na qualidade de vida, mas nem sempre um tempo de espera menor é melhor para o paciente ${ }^{11}$.

O tempo de espera por cirurgia eletiva varia segundo diversos fatores, que podem ser agrupados em duas categorias: relacionados à oferta de serviços, referentes à estrutura e processo, ou a características da demanda ${ }^{12}$. Entre os fatores estruturais encontramos na literatura: número de leitos disponíveis ${ }^{13}$; menor experiência do cirurgião e sua equipe em termos de volume de trabalho pregresso ${ }^{14}$; tipo de hospital ${ }^{9}$; e capacidade da rede pública ${ }^{5}$. Entre os fatores relativos ao processo, temos: cancelamento de eletivas devido à ocupação de leitos por urgências'; organização do hospital, eficiência do administrador e do gerenciamento hospitalar ${ }^{15}$; formação de redes hospitalares para o atendimento da demanda ${ }^{16}$; política de cobertura populacional e de procedimentos ${ }^{17}$; critérios de priorização de pacientes com indicações de urgência no procedimento ou em melhores condições clínicas, havendo grande concordância entre cirurgiões e clínicos (especialistas ou generalistas) quanto a estes dois últimos critérios ${ }^{18}$.

Ao conjunto de fatores relacionados à oferta, que fazem parte do modelo de atenção à saúde ${ }^{19}$, tem sido atribuída maior força na determinação do tempo de espera do que àqueles relacionados à demanda. Diferentes países possuem modelos de atenção distintos, daí a grande dificuldade em se fazer comparações internacionais.

Quanto aos fatores relacionados à demanda, encontramos na literatura: adiamento por medo da cirurgia ${ }^{20}$; condições socioeconômicas ${ }^{21}$; quantidade de procedimentos pré-operatórios ${ }^{20}$; sexo e idade $\mathrm{e}^{14}$; o tempo julgado adequado segundo a demanda, que é influenciado por gravidade e tipo de problema ${ }^{22}$; preferência por determinado cirurgião ou serviço ${ }^{23}$.

Como resultado, o tempo de espera descrito na literatura varia enormemente. Em nosso meio o estudo de diferenças no tempo de espera para realização de cirurgia eletiva seria de fundamental importância na verificação da situação da preconizada eqüidade horizontal.

O objetivo do presente trabalho é analisar possíveis diferenças no tempo de espera para uma cirurgia eletiva, comparando pacientes de clientela pública e privada em município de grande porte que disponha de uma rede de atenção à saúde de alta complexidade, e reproduza as características do Sistema de Saúde no Brasil onde há o Sistema Único de Saúde (SUS) e o Sistema Suplementar, que possuem formas de operacionalização diferentes, pois um é regido pelo poder público e o outro pelo poder privado.

A comparação entre hospitais representativos desses dois distintos compartimentos, público e privado, que se enquadram no nível de atenção terciária à saúde sendo referências para a rede de serviços de saúde, e concentram tecnologia compatível com seu porte ${ }^{19}$, é muito oportuna para o estudo do tema eqüidade, porque possuem 
clientelas com diferenças socioeconômicas importantes.

\section{Metodologia}

Realizou-se um estudo transversal de base hospitalar, com seleção de participantes segundo definição prévia de exposição. O hospital privado selecionado é de médio porte, realiza procedimentos de média e alta complexidade, atende exclusivamente clientela privada e pertence a uma cooperativa de médicos. O hospital público selecionado é de grande porte, realiza procedimentos de média e alta complexidade, atende exclusivamente clientela SUS, pertence à Secretaria de Estado da Saúde de São Paulo e é hospital-escola. Ambos estão localizados em Sorocaba (Tabela 1).

Em razão dos limites operacionais, foi definida uma amostra de conveniência de 40 pacientes para cada hospital, o que garante um poder de 0,80 para testar uma diferença no tempo de espera por cirurgia eletiva entre hospitais igual a 0,63 desvio padrão ${ }^{24}$.

Foram incluídos no estudo pacientes internados, no período pós-cirúrgico, submetidos à cirurgia eletiva, maiores de 18 anos de idade, e que comprovaram o tempo de espera para a realização da cirurgia.

A fim de viabilizar a comparabilidade entre os hospitais, selecionaram-se os tipos de cirurgia existentes entre as mais freqüentes nos dois hospitais. O hospital público não disponibilizou dados das cirurgias que ocorriam, o que obrigou à pré-análise com acompanhamento diário de todas as cirurgias eletivas que eram realizadas neste hospital no período de agosto e setembro de 2005. Diferentemente ocorreu no hospital privado, onde foi disponibilizada uma lista com o número de cirurgias realizadas no ano anterior, acrescido do acesso aos boletins com toda programação prévia das cirurgias a serem realizadas na semana.

Para o recrutamento e as entrevistas, durante os meses de outubro e novembro de 2005, o hospital público foi visitado diariamente e o hospital privado nos dias em que ocorreram as cirurgias de interesse, até se obter o número necessário de pacientes.

Nos dias das visitas identificavam-se os pacientes que eram elegíveis para o estudo e se procedia à entrevista com todos eles, via aplicação de questionário estruturado, que incluía informações sobre sexo, idade, escolaridade, renda familiar per capita, situação conjugal, procedência, tipo de cirurgia realizada, comorbidade (incluindo diabetes e/ou hipertensão), e tempo de espera para realização de cirurgia eletiva.

O tempo de espera utilizado na pesquisa, medido em número mínimo de meses completados e sendo mês igual a 30 dias, foi o período compreendido entre o diag-

Tabela 1 - Indicadores selecionados dos hospitais incluídos na pesquisa

Table 1 - Selected indicators from hospitals studied

\begin{tabular}{lcc}
\hline Hospital & Público & Privado \\
\hline Total de leitos operacionais & 326 & 104 \\
Média mensal de internações & 1063 & 951 \\
Índice de Giro* (Internações/Leitos) & 3,3 & 9,1 \\
Média mensal de cirurgias & 441 & 1039 \\
Média mensal de cirurgias eletivas & 233 & 516 \\
\% de cirurgias eletivas/total de cirurgias & 52,8 & 49,7 \\
\% de cirurgias eletivas/total de internações & 21,9 & 54,3 \\
\hline Fontes/Sources: Serviço de Arquivo Médico e Estatística (SAME) do Hospital UNIMED-Sorocaba e \\
do Conjunto Hospitalar de Sorocaba, 2005 \\
*Adaptado do original que é igual ao número de saídas / número de leitos \\
*Adapted from the original which is equal to the number of discharges / number of beds
\end{tabular}


nóstico definitivo da doença, clínico ou laboratorial, com a respectiva indicação da cirurgia, comprovado por guia de referência ou relatório médico com a solicitação do agendamento da referida cirurgia, e a data da realização da cirurgia.

A análise estatística incluiu o estudo da distribuição de freqüência das variáveis, testes de associação qui-quadrado ou exato de Fisher, testes das diferenças entre médias ANOVA, Mann-Whitney ou Kruskal-Wallis, e Correlação de Spearman.

Para a análise do efeito independente do tipo de hospital sobre o tempo de espera foram empregados modelos de regressão múltipla não paramétrica, método Least Absolute Value - LAV, nos quais foi incluída uma terceira variável selecionada entre as variáveis de confusão que apresentaram associação $(\mathrm{p}<0,20)$ com tempo de espera e tipo de hospital, testando-se uma por vez para evitar saturação do modelo ${ }^{25}$. Foi utilizado o pacote estatístico Stata ${ }^{\mathrm{tm} 26}$.

Este projeto foi aprovado pelo do Comitê de Ética em Pesquisa do CCMB/PUC-SP e pelos Comitês de Ética Médica de ambos os hospitais, e financiado pelo CNPq-PIBICPUCSP.

\section{Resultados}

Não houve recusa à participação nem perda de informação. Todas as perguntas foram respondidas na íntegra, não havendo dados ignorados. Todos os pacientes elegíveis recrutados comprovaram o tempo de espera.

Tratamento cirúrgico para varizes, colecistectomia, hemorroidectomia e histerectomia foram as cirurgias eletivas incluídas no estudo, porque estavam entre as mais freqüentes em ambos os hospitais. Representavam 15\% do total de eletivas. Observa-se na Tabela 1 que o hospital público apresenta um Índice de Giro $^{27}$ mensal, definido como 'número de internações dividido por número de leitos', bem menor $(3,3$ VS 9,1) e uma proporção menor de cirurgias eletivas sobre o total de internações (21,9 VS 54,3\%).

Na Tabela 2 descrevem-se as variáveis pesquisadas segundo o local de coleta das amostras (hospital público ou privado). Observam-se diferenças significantes para algumas variáveis sócio-econômicas, sendo no hospital privado maior o grau de escolaridade, maior a renda, e menor a proporção com procedência de outros municípios.

Na Tabela 3 é analisada a relação do tempo de espera com as variáveis pesquisadas. Verifica-se um tempo de espera significantemente maior para as seguintes variáveis/ categorias: hospital público (diferença de 5,5 meses, $\mathrm{p}<0,001$ ); menor escolaridade (diferença de 3,5 meses, $\mathrm{p}=0,01$ ); menor renda (coeficiente de Spearman $=-0,4426$, $\mathrm{p}<0,001$ ); procedência/outro município (diferença de 2 meses, p=0,009); diagnóstico / cirurgia de varizes (diferença de 7 meses comparada à colecistectomia, $\mathrm{p}=0,04$ para o conjunto de categorias).

Como potenciais variáveis de confusão, temos comorbidade, renda, escolaridade e procedência. Por meio da regressão múltipla para a relação entre tempo de espera e hospital, verifica-se que se mantém significante o efeito da variável tipo de hospital, sendo que o tempo de espera no hospital público é 5 meses maior quando ajustado por escolaridade ou procedência; 4,93 meses maior quando ajustado por renda, e 6 meses maior quando ajustado por comorbidade. Observa-se que renda, escolaridade ou procedência, quando ajustadas para tipo de hospital, deixam de apresentar diferenças significantes no tempo de espera. E a diferença segundo comorbidade permanece não significante (Tabela 4).

\section{Discussão}

Neste trabalho verifica-se que os pacientes do hospital público, quando comparados aos pacientes do hospital privado, apresentam maior tempo de espera para realizar determinadas cirurgias eletivas. Mostra-se uma discrepância muito acentuada que pode revelar elevado grau de iniqüidade relacionado ao tipo de prestador de serviços de saúde, e que se mantém significante mesmo quando ajustado por 
Tabela 2 - Descrição das amostras segundo as variáveis pesquisadas. Sorocaba, 2005.

Table 2 - Description of samples according to variables studied. Sorocaba, 2005.

\begin{tabular}{|c|c|c|c|c|c|c|}
\hline \multirow[t]{3}{*}{ Variáveis } & \multirow[t]{3}{*}{ Categorias/ Descritores } & \multicolumn{4}{|c|}{ Hospital } & \multirow[t]{3}{*}{$p$} \\
\hline & & \multicolumn{2}{|c|}{ Público } & \multicolumn{2}{|c|}{ Privado } & \\
\hline & & $\mathrm{N}$ & $\%$ & $\mathrm{~N}$ & $\%$ & \\
\hline \multirow[t]{3}{*}{ Sexo } & & & & & & $0,43^{*}$ \\
\hline & Feminino & 29 & 72,5 & 32 & 80 & \\
\hline & Masculino & 11 & 27,5 & 8 & 20 & \\
\hline \multirow[t]{3}{*}{ Escolaridade } & & & & & & $<0,001^{*}$ \\
\hline & $\begin{array}{l}\text { Até } 8^{a} \text { série do Ensino } \\
\text { Fundamental }\end{array}$ & 29 & 72,5 & 7 & 17,5 & \\
\hline & Ensino médio ou superior & 11 & 27,5 & 33 & 82,5 & \\
\hline \multirow[t]{6}{*}{ Situação conjugal } & & & & & & $0,33^{* *}$ \\
\hline & Solteiro & 10 & 25 & 7 & 17,5 & \\
\hline & Casado & 25 & 62,5 & 26 & 65 & \\
\hline & Divorciado & 0 & 0 & 3 & 7,5 & \\
\hline & Viúvo & 5 & 12,5 & 3 & 7,5 & \\
\hline & União informal & 0 & 0 & 1 & 2,5 & \\
\hline \multirow[t]{5}{*}{ Cirurgia realizada } & & & & & & $0,97^{*}$ \\
\hline & Safenectomia & 6 & 15 & 6 & 15 & \\
\hline & Colecistectomia & 17 & 42,5 & 15 & 37,5 & \\
\hline & Hemorroidectomia & 5 & 12,5 & 6 & 15 & \\
\hline & Histerectomia & 12 & 30 & 13 & 32,5 & \\
\hline \multirow[t]{3}{*}{ Procedência } & & & & & & $<0,001^{*}$ \\
\hline & Sorocaba & 9 & 22,5 & 27 & 67,5 & \\
\hline & Outros municípios & 31 & 77,5 & 13 & 32,5 & \\
\hline \multirow[t]{3}{*}{ Comorbidade } & & & & & & $0,13^{*}$ \\
\hline & Presença & 14 & 35 & 8 & 20 & \\
\hline & Ausência & 26 & 65 & 32 & 80 & \\
\hline \multirow[t]{3}{*}{ Idade(anos) } & & & & & & $0,17^{* * *}$ \\
\hline & Média & 49,75 & & 44,35 & & \\
\hline & Desvio padrão & 13,52 & & 15,34 & & \\
\hline \multirow[t]{3}{*}{ Renda(Reais) } & & & & & & $<0,001^{* * * *}$ \\
\hline & Mediana & 240 & & 1000 & & \\
\hline & p25-p75 & $150-325$ & & $562,50-1500$ & & \\
\hline
\end{tabular}

${ }^{*}$ Qui-quadrado **Exato de Fisher ${ }^{* * *}$ ANOVA ****Mann-Whitney

${ }^{*}$ Chi Square ${ }^{* *}$ Fisher's Exact ${ }^{* * *}$ ANOVA ${ }^{* * * *}$ Mann-Whitney

potenciais variáveis de confusão.

O tempo de espera também foi maior para as categorias de menor escolaridade, de menor renda, procedência de outros municípios e safenectomia. Porém, dentre as limitações decorrentes do pequeno tamanho da amostra, não há poder estatístico suficiente para a análise do efeito independente destas variáveis. Outra limitação que deve ser considerada é a mensuração da 
Tabela 3 - Tempo de espera (meses) segundo as variáveis pesquisadas. Sorocaba, 2005.

Table 3 - Waiting time (months) according to variables studied. Sorocaba, 2005.

\begin{tabular}{|c|c|c|c|c|}
\hline \multirow[t]{2}{*}{ Variáveis } & \multirow[t]{2}{*}{ Categorias } & \multicolumn{2}{|c|}{ Descritores } & \multirow[t]{2}{*}{$p$} \\
\hline & & Mediana & p25-p75 & \\
\hline \multirow[t]{3}{*}{ Hospital } & & & & $<0,001^{*}$ \\
\hline & Público & 7 & $3-13$ & \\
\hline & Privado & 1,5 & $1-2,5$ & \\
\hline \multirow[t]{3}{*}{ Sexo } & & & & $0,07^{*}$ \\
\hline & Feminino & 4 & $1-8$ & \\
\hline & Masculino & 2 & $1-3$ & \\
\hline \multirow[t]{3}{*}{ Escolaridade } & & & & $<0,001^{*}$ \\
\hline & $\begin{array}{l}\text { Até } 8^{a} \text { série do Ensino } \\
\text { Fundamental (EF) }\end{array}$ & 5,5 & $2-10,5$ & \\
\hline & $\begin{array}{l}\text { Ensino médio ou } \\
\text { superior }\end{array}$ & 2 & $1-3,75$ & \\
\hline \multirow[t]{6}{*}{ Situação conjugal } & & & $0,4^{* *}$ & \\
\hline & Solteiro & 6 & $2-14$ & \\
\hline & Casado & 3 & $1-7$ & \\
\hline & Divorciado & 1 & $1-9$ & \\
\hline & Viúvo & 3 & $1-5,5$ & \\
\hline & União informal & 2 & $2-2$ & \\
\hline
\end{tabular}

Procedência

$0,009^{*}$

$\begin{array}{lll}\text { Sorocaba } & 2 & 1-5,5 \\ \text { Outras } & 4 & 2-8,5\end{array}$

Comorbidade

$2-8,5$

$\begin{array}{lcc}\text { Presença } & 5,5 & 2-8\end{array}$

Cirurgia realizada

Ausência

$2 \quad 1-7$

\begin{tabular}{llccc} 
& Safenectomia & 9 & $3-21$ & \\
& Colecistectomia & 2 & $1-6,5$ & \\
& Hemorroidectomia & 2 & $1-4$ & \\
& Histerectomia & 4 & $1-7$ & \\
\hline Idade & Coeficiente de Spearman & \\
Renda & & & 0,8641 \\
& & $-0,0194$ & $<0,001$ \\
\hline
\end{tabular}

* Mann-Whitney ${ }^{* *}$ Kruskal-Wallis

variável tempo de espera em meses, que implica em menor precisão e menor poder para a verificação de eventuais diferenças.
Löfvendahl e cols. ${ }^{9}$ também encontraram maior tempo de espera para os hospitais universitários ou regionais na Suécia, sendo 
Tabela 4 - Diferenças no tempo de espera segundo tipo de hospital ajustadas para as potenciais variáveis de confusão (regressão múltipla não paramétrica)

Table 4 - Waiting time differences according to hospital type adjusted for potential confounding variables (non-parametric multiple regression)

\begin{tabular}{|c|c|c|c|c|c|c|}
\hline Modelo & Variável & Categorias & $\mathrm{b}$ (meses) & IC95\% & $\mathrm{p}$ & Pseudo $\mathrm{R}^{2}$ \\
\hline \multirow[t]{3}{*}{1} & & & & & & 0,1973 \\
\hline & Hospital & Público & 5,00 & 2,7 a 7,3 & $<0,001$ & \\
\hline & Procedência & Outros municípios & 1,00 & $-1,2$ a 3,2 & 0,38 & \\
\hline \multirow[t]{3}{*}{2} & & & & & & 0,1868 \\
\hline & Hospital & Público & 4,93 & 3.4 a 6.4 & $<0,001$ & \\
\hline & Renda & Reais & $-0,0003$ & $-0,001$ a 0,0004 & 0,420 & \\
\hline \multirow[t]{3}{*}{3} & & & & & & 0,1918 \\
\hline & Hospital & Público & 5,00 & 2,5 a 7,5 & $<0,001$ & \\
\hline & Escolaridade & Até 8a ${ }^{a}$.série EF & 1,00 & $-1,5$ a 3,0 & 0,420 & \\
\hline \multirow[t]{3}{*}{4} & & & & & & 0,1918 \\
\hline & Hospital & Público & 6,00 & 4,0 a 8,0 & $<0,001$ & \\
\hline & Comorbidade & Presença & 1,00 & $-1,4$ a 3,4 & 0,403 & \\
\hline
\end{tabular}

o tipo de hospital o fator estudado com maior magnitude de efeito e significância. Segundo estes autores, a possível explicação seria o cancelamento mais freqüente de eletivas nestes hospitais devido aos casos, sobretudo clínicos, de emergência. Apesar de se tratar de um modelo de atenção à saúde diferente do modelo brasileiro, esta explicação é plausível nesta amostra, pois o hospital público incluído dispõe de uma unidade regional de emergência que é referência para 45 municípios de uma área com aproximadamente 2 milhões de habitantes, e apresenta uma proporção de cirurgias eletivas em relação ao total de internações bem menor que o hospital privado.

Vários autores defendem medidas organizacionais específicas para a garantia das cirurgias eletivas: administrá-las separadamente, gerenciamento especifico da lista de espera, reestruturação das sessões de cirurgia, planejamento de altas mais precoces ${ }^{8,15}$. Dawson e cols. ${ }^{16}$ propõem a formação de redes hospitalares de apoio mútuo, de modo a oferecer aos pacientes a possibilidade de escolha por hospitais cuja espera seja menor, o que na sua experiência, em Londres, reduziu o tempo de espera em 3-4 semanas .
Mas outras possíveis explicações para o maior tempo de espera verificado no hospital público, e que caracterizam parte significativa dos serviços públicos de saúde, devem ser incluídas: longa fila de espera para realização de consultas, exames ou cirurgias; falta de médicos e funcionários; grande demora nas referências e no agendamento para outros serviços; profissionais muitas vezes pouco preparados para o atendimento; condições de trabalho e de remuneração incompatíveis com a grande demanda; e orçamento público insuficiente $^{28,29}$. Entretanto, Duckett ${ }^{5}$ defende a importância do hospital público como regulador do sistema, tendo verificado, na Austrália, que a mediana do tempo de espera apresenta relação inversa com a proporção de atendimento público.

Em complemento, prestadores públicos e privados têm clientelas diferenciadas no que se refere ao perfil de renda. A diferença no tempo de espera segundo a renda traduz iniqüidade referente à questão socioeconômica, diretamente relacionada ao poder de compra dos serviços de saúde, e que tem sido observada em vários estudos ${ }^{21}$. Também suas clientelas são diferenciadas quanto à origem, evidenciando maior difi- 
culdade de acesso àqueles procedentes de outros municípios da região, mais uma face da iniqüidade na atenção à saúde. Damiani e cols. ${ }^{13}$ analisando onde a demanda excedia 6 meses de espera, no Reino Unido, encontrou associação com a menor disponibilidade de leitos, que variava geograficamente e implicava em dificuldade de acesso e longas viagens para obter cuidados.

Outro aspecto a ser discutido refere-se aos diagnósticos e à comparação entre os procedimentos. Na literatura encontramos grande variabilidade no tempo de espera segundo o tipo de cirurgia.

Em Pernambuco observaram ${ }^{20}$ média de 1,1 mês para cirurgia de catarata, e em Sergipe $^{30} 6,28$ meses para transplante de córnea.

Na Nova Zelândia, MacCormick e Parry ${ }^{31}$ encontraram variação na média do tempo de espera de 42 dias para cirurgias de câncer a 317 dias para herniorrafia, sendo de 250 dias para hemorroidectomia e 173 dias para colelitíase, espera que, para os dois últimos tipos de cirurgia, é bem maior que a encontrada no presente estudo, igual a 2 meses, destacando um aspecto positivo do nosso sistema de atenção à saúde.

Oudhoff e cols. ${ }^{18}$ defendem um tempo de espera aceitável entre 2 e 25 semanas, dependendo do tipo e gravidade do problema. Estes autores encontraram, na Holanda, uma variação de 98 dias para casos de nefrolitíase a 170 dias para casos de varizes, este último com resultado menor que o encontrado na presente pesquisa, de 9 meses.

Diante de tal variabilidade no tempo de espera, deve-se considerar que o procedimento de selecionar alguns tipos de cirurgia, adotado neste trabalho, resultou em importante limitação, podendo ter atenuado as diferenças no tempo de espera entre os hospitais.

Acrescente-se a possibilidade de existirem diferentes perfis de gravidade e de condições cirúrgicas entre as clientelas, variáveis que podem não ter sido captadas, cuja dimensão é bem maior do que a investigação efetivamente realizada nesta pesquisa sobre comorbidade, restrita à hipertensão e ao diabetes ${ }^{18}$. Em outras palavras, o hospital público pode assistir a um conjunto de pacientes mais críticos e que provavelmente apresentam prevenção primordial precária, pois são expostos a maior número de fatores de risco estruturais, por exemplo, o desemprego. Some-se prevenção primária mais falha tanto na promoção da saúde quanto na proteção específica, por exemplo, hábitos saudáveis e falhas na prevenção secundária com diagnóstico tardio e tratamento insatisfatório, e têm-se um paciente de risco e com prognóstico comprometido, gerando a necessidade de melhorar a condição clínica geral do paciente para tê-lo em condições de cirurgia. O Índice de Giro bem menor no hospital público parece corroborar com estas suposições.

Deste modo, o paciente do prestador público apresentando uma situação de vida mais difícil acaba tendo pior aderência aos requisitos pré-operatórios, que pode se aliar de modo perverso à menor eficiência da rede para $\mathrm{o}$ atendimento destes requisitos. Lima et cols. ${ }^{20}$ encontraram relação direta entre o aumento do tempo de espera e o maior número de vezes que o paciente deve comparecer ao serviço de saúde na etapa pré-operatória.

Não apenas melhor condição clínica explica menor tempo de espera. A decisão de ser operado pode ser mais objetiva na clientela do prestador privado, podendo-se cogitar inclusive dos fatores qualidade do vínculo e confiança com o profissional e o serviço, enquanto o paciente do prestador público, por insegurança, protela a decisão e prefere continuar apenas com o tratamento clínico enquanto for possível. Na literatura encontramos, na opinião dos pacientes, o medo como motivo para o adiamento da cirurgia $^{20}$, e a maior gravidade motivo para sua antecipação ${ }^{22}$.

A eqüidade horizontal, entendida como eqüidade intranecessidades, implica no principio de igualdade de acesso e tem sido operacionalmente tratado como igualdade de oportunidade na utilização de serviços de saúde ou igualdade de tratamento para 
necessidades iguais. Indivíduos portadores de um mesmo problema de saúde, independentemente de sua condição social e econômica, devem ter a mesma oportunidade de utilizar serviços de saúde e receber cuidados médicos adequados ${ }^{32}$.

No Brasil há uma mistura entre as teorias normativas de eqüidade, pois coexiste tanto a via liberal, simbolizada pelo modelo americano com menor envolvimento do Estado, quanto a via igualitária, muito freqüente na Europa ${ }^{19}$. Esta falta de consenso resulta num modelo de atenção misto com seguros privados de saúde, suplementares ao SUS, e pode se tornar um obstáculo ideológico à eqüidade ${ }^{17}$.

Em conclusão, verifica-se maior tempo de espera na clientela do hospital público para o grupo de cirurgias eletivas estudadas. As implicações do atraso para a realização de uma cirurgia eletiva incluem além dos problemas físicos, problemas psicológicos e sociais ${ }^{9}$, resultando em pior qualidade de vida ${ }^{10}$.

Enfim, o presente estudo contribui para tornar mais clara a necessidade premente da aplicação de um conjunto de medidas referentes à política de saúde para atenuar essa situação de iniqüidade.

\section{Referências}

1. Rouquayrol MZ, Almeida-Filho N. Epidemiologia e Saúde. Rio de Janeiro: MEDSI; 2003.

2. Pereira J. Glossário de economia da saúde. In: Piola SF, Viana SM. Economia da Saúde: conceito e contribuição para a gestão da saúde. Brasília: IPEA; 1995. p. 271-93.

3. Brasil. Ministério da Saúde. Secretaria Técnica da RIPSA. Indicadores e dados básicos para saúde. Brasília: Ministério da Saúde; 2001.

4. Organización Mundial de la Salud. Preparación de indicadores para vigilar los progressos realizados en el logro de la salud para todos en el año 2000. Ginebra: OMS; 1981.

5. Duckett SJ. Finance and Policy: Private care and public waiting. Aust Health Rev 2005; 29 (1): 87-93.

6. Organización Mundial de la Salud. La salud en las Américas. Washington, DC: OPS; 1998.

7. Moraes NLA. Níveis de Saúde de coletividades brasileiras. Rev SESP 1999; 2: 403-97.

8. Robb WB, O’Sullivan MJ, Brannigan AE, Bouchier-Hayes DJ. Are elective surgical operations cancelled due to increasing medical admissions? Ir J Med Sci 2004; 173(3): 129-32.

9. Löfvendahl S, Eckerlund I, Hansag H, Malmqvist B, Resch S, Hanning M. Waiting for orthopaedic surgery: factors associated with waiting times and patients' opinion. Int J Qual Health Care 2005; 17(2): 133-40.

10. Lawrentshuk N, Hewitt PM, Pritchard MG. Elective Laparoscopic Cholecystectomy: implications of prolonged waiting times for surgery. ANZ J Surg 2003; 73: 890-3.
11. Chouillard E et al. Colectomía laparoscópica programada en la diverticulitis no complicada: ¿cuándo se debe intervenir quirúrgicamente? Cir Esp 2007; 81(4): 207-12.

12. Midttun L, Martinussen PE. Hospital waiting time in Norway: What is the role of organizational change? Scand J Public Health 2005; 33(6): 439-46.

13. Damiani M, Propper C, Dixon J. Mapping choice in the NHS: cross sectional study of routinely collected data. BMJ 2005; 330(7486): 284

14. Lau R, Vair BA, Porter GA. Factors influencing waiting times for elective laparoscopic cholecystectomy. Can J Surg 2007; 50(1): 34-8.

15. Singh N, Brooke-Cowden GL, Whitehurst C, Smith D, Senior J. The Auburn Elective Surgery Pilot Project. ANZ J Surg 2005; 75: 768-75.

16. Dawson D, Gravelle H, Jacobs R, Martin S, Smith PC. The effects of expanding patient choice of provider on waiting times: evidence from a policy experiment. Health Econ 2007; 16: 113-28.

17. Gianini RJ, Traynor M. Questões ideológicas de eqüidade na atenção à saúde no Brasil: um estudo do período 1993 - 2000. Rev Fac Ciênc Méd Sorocaba 2006; 8(2): 11 17.

18. Oudhoff JP, Timmermans DR, Rietberg M, Knol DL, Van der Wal G. The acceptability of waiting times for elective general surgery and the appropriateness of prioritising patients. BMC Health Serv Res 2007; 28(7): 32.

19. Paim JS, Almeida-Filho N. Saúde coletiva: uma nova saúde pública ou campo aberto a novos paradigmas? Rev Saúde Pública 1998; 32(4): 299-316. 
20. Lima DMG, Ventura LO, Brandt CT. Barreiras para o acesso ao tratamento da catarata senil na Fundação Altino Ventura. Arq Bras Oftalmol 2005; 68(3): 357-62.

21. Shortt SED, Shaw RA. Equity in Canadian health care: Does socioeconomic status affect waiting times for elective surgery? CMAJ 2003; 168(4): 413-6.

22. Mariano ER, Chu LF, Ramamoorthy C, Macario A. Scheduling Elective Pediatric Procedures That Require Anesthesia: The Perspective of Parents. Pediatric Anesthesia 2006; 103(6): 1426-31.

23. Cromwell DA. Social waiting time information services: An evaluation of how well clearance time statistics can forecast a patient's wait. Science \& Medicine 2004; 59: 1937-48.

24. Rosner B. Fundamentals of Biostatistics. Boston: Duxbury Press; 1990.

25. Katz MH. Multivariable Analysis: A Primer for Readers of Medical Research. Ann Intern Med 2003; 138: 644-50.

26. StataCorp. Stata ${ }^{\text {tm }}$ 9.0: Statistics/Data analysis. Texas: StataCorp; 2005.

27. Zucchi P, Bittar OJNV, Haddad N. Produtividade em hospitais de ensino no estado de São Paulo, Brasil. Rev Panam Salud Publica 1998; 4(5): 311-6
28. Sobolev B, Brown P, Zelt D, Kuramoto L. Waiting time in relation to wait-list size at registration: statistical analysis of a waiting-list registry. Clin Invest Med 2004; 27(6): 298-305.

29. Gouvea CSD, Travassos C, Fernandes C. Produção de serviços e qualidade da assistência hospitalar no Estado do Rio de Janeiro, Brasil - 1992 a 1995. Rev Saúde Pública 1997; 31(6): 601-17.

30. Araújo AA, Melo GB, Silva RL, Araújo-Neta VM. Perfil epidemiológico dos pacientes na lista de espera para transplante de córnea no Estado de Sergipe Arq Bras Oftalmol 2004; 67(4): 613-6.

31. MacCormick AD, Parry BR. Waiting time threshold: are they appropriate? ANZ J Surg 2003; 73: 926-8.

32. Travassos C. Equidade e o Sistema Único de Saúde: uma contribuição para debate. Cad Saúde Publica 1997; 13(2): 325-30.

Recebido em: 11/10/07

Versão final reapresentada em: 30/06/08 Aprovado em: 16/07/08 